\title{
Effective apsidal precession from a monopole solution in a Zipoy spacetime
}

\author{
Abraão J. S. Capistrano ${ }^{1, \mathrm{a}}$, Paola T. Z. Seidel ${ }^{1, \mathrm{~b}}$, Luís A. Cabral ${ }^{2, \mathrm{c}}$ \\ ${ }^{1}$ Applied Physics Graduation Program, UNILA, Foz do Iguaçu, PR 85867-670, Brazil \\ ${ }^{2}$ Universidade Federal do Tocantins, Curso de Física, Setor Cimba, Araguaína, TO 77824-838, Brazil
}

Received: 11 July 2019 / Accepted: 20 August 2019 / Published online: 30 August 2019

(C) The Author(s) 2019

\begin{abstract}
In this work, we examine the orbit equations originated from Zipoy's oblate metric. Accordingly, the solution of Einstein's vacuum equations can be written as a linear combination of Legendre polynomials of positive definite integers $l$. Starting from the zeroth order $l=0$, in a nearly newtonian regime, we obtain a non-trivial formula favoring both retrograde and advanced solutions for the apsidal precession, depending on parameters related to the metric coefficients. Using a Chi-squared statistics, we apply the model to the apsidal precessions of Mercury and asteroids (1566 Icarus and 2-Pallas). As a result, we show that the obtained values favor the oblate solution as a more adapted approach as compared to those results produced by Weyl's cylindric and Schwarzschild solutions. Moreover, it is also shown that the resulting solution converges to the integrable case $\gamma=1$ in the sense of the Zipoy-Voorhees metric.
\end{abstract}

\section{Introduction}

Since the explanation of the perihelion advance of Mercury by Einstein in 1915 as an application of general Relativity (GR), it has been considered one of the fundamental laboratories for testing extensions of standard GR and other gravitational models such as e.g, the modification of newtonian Dynamics (MOND)[2], Kaluza-Klein five-dimensional gravity [3], Yukawa-like Modified Gravity [4], HoravaLifshitz gravity [5], brane-world models and variants [612], the parametric post-newtonian (PPN) framework and beyond, and approaches in the weak field/slow motion limits [13-26]. As largely known, in GR, the coordinate systems are overall physically equivalents and to obtain new prospects on applications in astrophysics, the diffeomorphic

\footnotetext{
a e-mail: abecapistrano@gmail.com

b e-mail: paola.seidel@gmail.com

c e-mail: cabral@uft.edu.br
}

transformations cannot be allowed to happen. Hence, for any other solution of Einstein's, which is not diffeomorphic to the Schwarzschild's solution, it will produce a different nearly newtonian dynamics.

An interesting work published by Zipoy [27] investigates quasi-oblate spheroidal and prolate coordinates by calculating the vacuum Einstein's equations to study general properties of the metrics such as their topology, asymptotic behaviour, singularities and stability. Moreover, he found that those metrics present a nearly newtonian solution from a linear combination of Legendre polynomials. Bearing in mind that the astrophysical phenomena depend on the form of objects, different metrics must provide different aspects of the background physics of the phenomena in a lower gravitational field regime, as compared to the strong Einstein gravity. We use the term nearly newtonian in the sense of [28], and [29], as an intermediate strength of the gravitational field between GR and newtonian gravitational field in such a way that there is no constraints a priori on the field strength but only on the related movement (geodesic) equations. Needless to say, whenever the presuppositions of the weak field regime and the slow motion condition are applied and the expansion parameters of the metric are set, it leads naturally to the post-newtonian regime [30].

This paper also aims at investigating how different spacetimes may describe an astrophysical phenomenon with departure from a spherical geometry. In the second section, we make a brief review of Zipoy's work on oblate static metric and the "monopole" solution that resides on the zeroth degree of Legendre polynomials with a calculation of the related orbit equation. In the third section, the calculations of a nonstandard expression for the perihelion shift are shown with a comparison with the standard Einstein's result and Weyl's axial metric. We also apply the model to analyse the apsidal precession of the asteroids Icarus and 2-Pallas of the inner and outer solar system, respectively. Finally, we make the final remarks in the conclusion section. 


\section{Zipoy quasi-oblate metric}

\subsection{Form and general solution of Zipoy's metric}

We consider the effects in a single plane of orbits, which is compatible with the observed movement of the planets around the Sun limited roughly to the plane of their orbits. Considering the Sun in the center of the circular base of a cylinder and a planet (or a small celestial object) as a particle with mass $m$ orbiting its edge, it can be described by Weyl's line element [31]

$d s^{2}=-e^{2(\lambda-\sigma)}\left(d \rho^{2}+d z^{2}\right)-\rho^{2} e^{-2 \sigma} d \phi^{2}+e^{2 \sigma} d t^{2}$,

where the coefficients $\lambda=\lambda(\rho, z)$ and $\sigma=\sigma(\rho, z)$ are the Weyl potentials. This metric is diffeomorphic to the Schwarzschild's metric and is asymptotically flat [27,3134].

Differently from the works of [35-38] and [39], where the authors use a mass distribution to model galactic relativistic disks with Weyl's exact solution of Einstein equations, we investigated in [40] approximated solutions of this metric for a test particle in the perihelion precession by expanding the coefficient functions (or potentials) of the metric into a Taylor's series. As a result, e.g., we obtained the perihelion shift of Mercury about 43.105 arcsec/century in accordance with observations. Recently, an additional relativistic effect to the apsidal precession of Mercury was proposed as a result from "interacting terms" on the second-post-newtonian contribution [41] evincing that low-velocity limit regimes of GR is still an import arena of research in the realm of the astrophysical phenomena.

To obtain the quasi-oblate coordinates from Weyl coordinates, a change of variable can be applied in such a form $\rho=a \cosh v \cos \theta$ and $z=a \sinh v \sin \theta$, and $a$ is a length parameter. The resulting line element is given by

$$
\begin{aligned}
d s^{2}= & a^{2} e^{2(\lambda-\sigma)}\left(\sinh ^{2} v+\sin ^{2} \theta\right)\left(d v^{2}+d \theta^{2}\right) \\
& +a^{2} e^{-2 \sigma} \cosh ^{2} v \cos ^{2} \theta d \phi^{2}-e^{2 \sigma} d t^{2}
\end{aligned}
$$

where $(v, \theta)$ are the quasi-oblate coordinates. Variations of the coordinate $v$ produce ellipsoids intertwined by hyperboloids built with the coordinate $\theta$. Moreover, the exterior gravitational field is given by Einstein's vacuum equations

$$
\begin{aligned}
& \sigma_{, v v}+\sigma_{, \theta \theta}+\sigma_{, v} \tanh v-\sigma_{, \theta} \tan \theta=0, \\
& \sigma_{, v}^{2}-\sigma_{, \theta}^{2}-\lambda_{, v} \tanh v-\lambda_{, \theta} \tan \theta=0, \\
& 2 \sigma_{, v} \sigma_{, \theta}+\lambda_{, v} \tan \theta-\lambda_{, \theta} \tanh v=0, \\
& \lambda_{, v v}+\lambda_{, \theta \theta}+\sigma_{, v}^{2}+\sigma_{, \theta}^{2}=0 .
\end{aligned}
$$

where the notation $(, v),(, \theta)$ and $(, v v),(, \theta \theta)$ denote respectively the first and the second derivatives with respect to the variables $v$ and $\theta$. Noting that Eq. (3) is just Laplace's equation in oblate coordinates, a solution of the coefficient $\sigma$ can be found. Firstly, a change of variables can be made with $x=\sinh v$ and $y=\sin \theta$, and after using the method of separation of variables, one can write $\sigma(x, y)=P(x) Q(y)$, and find

$\frac{\partial}{\partial x}\left[\left(x^{2}+1\right) \frac{\partial \sigma}{\partial x}\right]+\frac{\partial}{\partial y}\left[\left(1-y^{2}\right) \frac{\partial \sigma}{\partial y}\right]=0$,

and their resulting separated equations

$$
\begin{aligned}
& \frac{\partial}{\partial x}\left[\left(x^{2}+1\right) \frac{\partial P(x)}{\partial x}\right]-l(l+1) P(x)=0, \\
& \frac{\partial}{\partial y}\left[\left(1-y^{2}\right) \frac{\partial Q(y)}{\partial y}\right]+l(l+1) Q(y)=0,
\end{aligned}
$$

where $l$ are the degree of Legendre polynomials. The solutions $P(x)$ and $Q(y)$ are given by the Legendre polynomials of first kind and both Legendre polynomials of first and (the complex) second kind, respectively. This set of equations and solutions were also discussed in [42-44]. Due to the structure of the line element in Eq. (2), we only need the coefficient $\sigma$ to produce a nearly newtonian gravitational regime by the component $g_{44}$ [28]. For this reason, we are only interested in the solution for the coefficient $\sigma$. Following the results in [27], for the "monopole" solution $l=0$, one can obtain:

$e^{2 v}=\left(\frac{r^{2}+a^{2} \sin \theta^{2}}{r^{2}+a^{2}}\right)^{\beta^{2}+1}$

and the $\sigma(r)$ potential is given by

$\sigma(r)=-\beta \arctan \frac{a}{r}$,

being $0 \leq \arctan \frac{a}{r} \leq \pi, \beta=\frac{m}{a}$ and $r=a \sinh v$. The quantities $a$ and $m$ are length parameters, being $\beta$ a dimensionless quantity. Hereon, we consider only $a$ and $\beta$ as fundamental parameters for our further analysis. This new change of variable leads to the line element

$$
\begin{aligned}
d s^{2}= & e^{2(v-\sigma)} d r^{2}+e^{2(v-\sigma)}\left(r^{2}+a^{2}\right) d \theta^{2} \\
& +e^{-2 \sigma}\left(r^{2}+a^{2}\right) \cos ^{2} \theta d \phi^{2}-e^{2 \sigma} d t^{2} .
\end{aligned}
$$

In this original work, Zipoy showed when $r \rightarrow \infty$, the Eq. (11) turns into an isotropic Schwarzschild line element and the set of coordinates $(r, \theta, \phi)$ turns the usual spherical coordinates.

We stress that the non-standard ingredient of this work is the space-time itself: rather than some deformation of a spherically symmetric field, we consider the "monopole" Zipoy's original metric vacuum solution as a model for the local solar-system gravitational field on test-particle orbiting its center. Hence, we do not use any energy-momentum tensor to propose a general relativistic disk-like model by using the Zipoy-Vorhees metric [27,45] which has been vastly 
explored in astrophysical literature [37-39] particularly for galaxy modelling. The Zipoy-Vorhees metric is referred to as $\gamma$-metric and the $\gamma$ parameter can be identified in Eq. (9) as $\gamma=\beta^{2}+1$. The two possible non-chaotic (integrable) solutions are when $\gamma$ is "nearly-minkowskian" $(\gamma \longrightarrow 0)$ or "nearly-Schwarzschildian" $(\gamma \longrightarrow 1)$. A larger discussion on Zipoy-Vorhees metric and variants can be found in $[46,47,56]$. It has been point out that the Zipoy-Vorhees metric are a form of the static limit of the Tomimatsu-Sato family of solutions $[48,49]$, but the underlying source of that metric, originally proposed by Voorhees, still remains an open problem. Moreover, Gibbons and Volkov [50] also explored the oblate Zipoy-Voorhees metric, rather than just a deformation of the Schwarzschild one, discussing the consequences of a ring wormhole. The properties of the $\gamma$-metric and in particular the motion of test particles have been investigated also in [51-59].

\subsection{Orbit equation for the "monopole" solution $l=0$}

The monopole solution of Zipoy's metric has a two-sheeted topology (involving two asymptotically flat regions) with both positive and negative $\theta$ and $r$ coordinates. In order to correspond to the distribution of matter in the known astrophysical systems (time-like trajectories), we restrain the $r$ coordinate to its positive values with the $\theta$ coordinate resigned to the plane of the orbits, since each sheet remains asymptotically Schwarzschil-dian, and the $g_{33}$ component is positive, there are no closed time-like curves. We consider a constraint to restrain the movement of a test-particle to the plane of the orbit setting the coordinate $\theta=0$. Hence, we have a constraint on velocities

$\mathbf{v} \cdot \mathbf{v}=g_{\alpha \beta} v^{\alpha} v^{\beta}=-1$,

where we denote $v^{\alpha}=\frac{d x^{\alpha}}{d \tau}$. Thus, we also denote the quantities $v^{r}=\frac{d r}{d \tau}, v^{\phi}=\frac{d \phi}{d \tau}$, and $v^{t}=\frac{d t}{d \tau}$. Moreover, using Eqs. (11) and (12), one can obtain the following expression

$$
\begin{aligned}
& -\left(\frac{r^{2}}{r^{2}+a^{2}}\right)^{\beta^{2}+1} e^{-2 \sigma(r)}\left(\frac{d r}{d \tau}\right)^{2} \\
& -e^{-2 \sigma(r)}\left(r^{2}+a^{2}\right)\left(\frac{d \phi}{d \tau}\right)^{2}+e^{2 \sigma(r)}\left(\frac{d t}{d \tau}\right)^{2}=-1 .
\end{aligned}
$$

To proceed further, we need to know the conserved quantities. This can be obtained using the Euler-Lagrange equations,

$$
\frac{\partial \mathcal{L}}{\partial x^{\mu}}-\frac{d}{d \tau}\left(\frac{\partial \mathcal{L}}{\partial \dot{x}^{\mu}}\right)=0
$$

where $\mathcal{L}$ is the Lagrangian functional commonly denoted as $\mathcal{L}=\frac{1}{2} g_{\mu \nu} \dot{x}^{\mu} \dot{x}^{\nu}$. For the interested case, we set the dependence of $\dot{x}^{\mu}$ for the coordinates $\phi$ and $t$. Hence, one finds

$$
\left(\frac{d \phi}{d \tau}\right)^{2}=\frac{L^{2} e^{4 \sigma(r)}}{\left(r^{2}+a^{2}\right)^{2}}
$$

and also

$$
\left(\frac{d t}{d \tau}\right)^{2}=E^{2} e^{-4 \sigma(r)}
$$

where we denote the conserved quantities $L$ for the specific orbital angular momentum and $E$ for the specific orbital energy. With those previous results, we can rewrite Eq. (13) in a form

$$
\begin{aligned}
& -\left(\frac{r^{2}}{r^{2}+a^{2}}\right)^{\beta^{2}+1} e^{-2 \sigma(r)}\left(\frac{d r}{d \tau}\right)^{2}-\left(\frac{L^{2} e^{2 \sigma(r)}}{r^{2}+a^{2}}\right) \\
& +e^{-2 \sigma(r)} E^{2}=-1,
\end{aligned}
$$

and after a little algebra, one finds

$$
\begin{aligned}
\left(\frac{d r}{d \phi}\right)^{2}= & {\left[1-\frac{L^{2} e^{2 \sigma(r)}}{\left(r^{2}+a^{2}\right)}+e^{-2 \sigma(r)} E^{2}\right] } \\
& \times \frac{e^{-2 \sigma(r)}}{L^{2}}\left(\frac{r^{2}+a^{2}}{r^{2}}\right)^{\beta^{2}+1}\left(r^{2}+a^{2}\right)^{2} .
\end{aligned}
$$

Taking a change of variable $u=\frac{1}{r}$, we can find an orbit equation

$$
\begin{aligned}
\left(\frac{d u}{d \phi}\right)^{2}= & -u^{2}\left(1+a^{2} u^{2}\right)^{\beta^{2}+2} \\
& +\frac{e^{-2 \sigma(u)}}{L^{2}}\left(1+a^{2} u^{2}\right)^{\beta^{2}+3}\left[1+E^{2} e^{-2 \sigma(u)}\right],
\end{aligned}
$$

and developing the previous equation, we have

$$
\begin{aligned}
& \left(\frac{d u}{d \phi}\right)^{2}=-u^{2}\left(1+2 a^{2} u^{2}+a^{4} u^{4}\right)\left(1+a^{2} u^{2}\right)^{\beta^{2}} \\
& +\frac{e^{-2 \sigma(u)} C(u)}{L^{2}}\left(1+a^{2} u^{2}+2 a^{2} u^{2}+2 a^{4} u^{4}\right)\left(1+a^{2} u^{2}\right)^{\beta^{2}} \\
& +\frac{e^{-2 \sigma(u)} C(u)}{L^{2}}\left(a^{4} u^{4}+a^{6} u^{6}\right)\left(1+a^{2} u^{2}\right)^{\beta^{2}}
\end{aligned}
$$

where we denote $C(u)=1+E^{2} e^{-2 \sigma(u)}$. Equivalently, we can write

$$
\begin{aligned}
\left(\frac{d u}{d \phi}\right)^{2}= & \alpha(u) u^{2}\left[\frac{3 a^{2} C(u)}{e^{2 \sigma(u)} L^{2}}-1\right] \\
& +\alpha(u) a^{2} u^{4}\left[\frac{3 a^{2} C(u)}{e^{2 \sigma(u)} L^{2}}-2\right]
\end{aligned}
$$




$$
\begin{aligned}
& +\alpha(u) a^{4} u^{6}\left[\frac{a^{2} C(u)}{e^{2 \sigma(u)} L^{2}}-1\right] \\
& +\frac{\alpha(u) C(u)}{e^{2 \sigma(u)} L^{2}},
\end{aligned}
$$

where we denote $\alpha(u)=\left(1+a^{2} u^{2}\right)^{\beta^{2}}$. Hence, a more convenient form for the resulting orbit equation can be written as

$$
\begin{aligned}
\left(\frac{d u}{d \phi}\right)^{2}= & \alpha(u) u^{2}\left[\frac{3 a^{2} C(u)}{e^{2 \sigma(u)} L^{2}}-1\right] \\
& +\alpha(u) a^{2} u^{4}\left[\frac{3 a^{2} C(u)}{e^{2 \sigma(u)} L^{2}}-2\right] \\
& +\alpha(u) a^{4} u^{6}\left[\frac{a^{2} C(u)}{e^{2 \sigma(u)} L^{2}}-1\right] \\
& +\frac{\alpha(u) C(u)}{e^{2 \sigma(u)} L^{2}} .
\end{aligned}
$$

It is noteworthy to point out that this equation is a highly nonlinear type, even in the simplest "monopole" case with $l=0$ and $\theta=0$.

\section{Analysis on apsidal precession}

To work with Eq. (22), we attenuate the field strength by analyzing the decaying terms and by the magnitude of the $\beta$ parameter, which is related to the coefficient $\sigma$ by Eq. (10). Firstly, we start truncating high orders of the variable $u$ constrained to $u^{4}$, since the effects $\mathcal{O}\left(u^{5}\right)$ in solar system scale are negligible [60]. Hence,

$$
\begin{aligned}
\left(\frac{d u}{d \phi}\right)^{2}= & \alpha(u) u^{2}\left[\frac{3 a^{2} C(u)}{e^{2 \sigma(u)} L^{2}}-1\right] \\
& +\alpha(u) a^{2} u^{4}\left[\frac{3 a^{2} C(u)}{e^{2 \sigma(u)} L^{2}}-2\right]+\frac{\alpha(u) C(u)}{e^{2 \sigma(u)} L^{2}} .
\end{aligned}
$$

Due to the fact that the previous orbit equation still remains strongly nonlinear, a general $\beta$ parameter on $\alpha(u)$ compromises the integrability of the equations of motion, which makes unpracticable to get any closed analytic solution. We can study approximate solutions if we impose that the parameter $\beta$ is small, then the length parameter $a$ must be large. Moreover, for small values of the $\beta$ parameter, the term $\alpha(u)$ can be expanded as $\alpha(u)=1+\beta^{2} a^{2} u^{2}+O(u)^{4}$. Clearly, the third order will produce terms of orders higher than $u^{4}$ in the main equation in Eq. (23), so the expansion in the term $\alpha(u)$ is truncated up to $u^{2}$. On the other hand, since $E$ should be the specific orbital energy, from the term $C(u)$ we find that $E^{2} e^{-2 \sigma(u)}>>1$. These two considerations lead us to a more treatable orbit equation in such a form
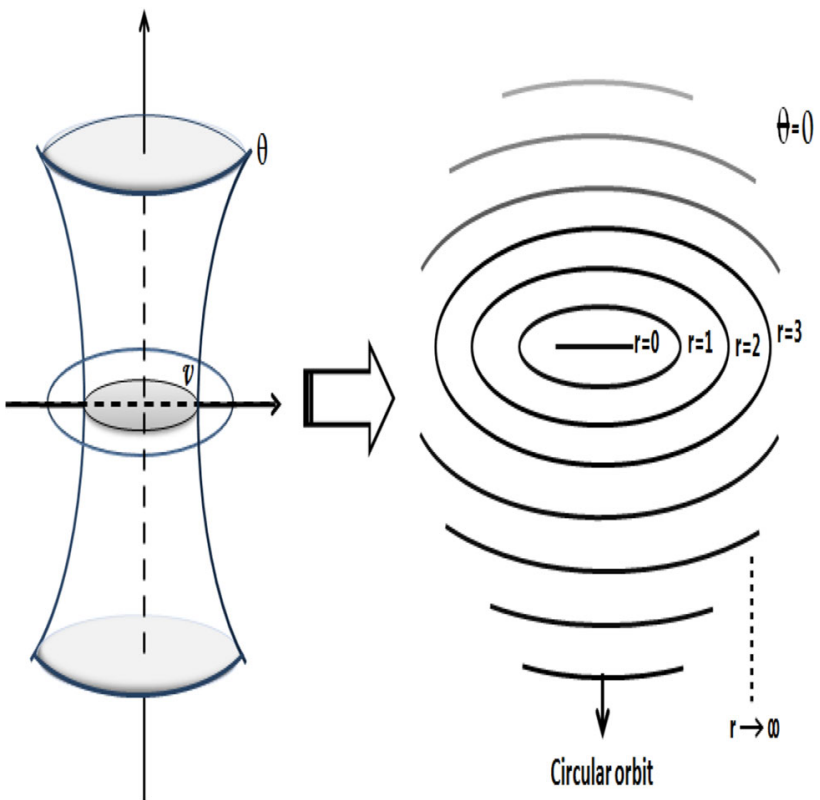

Fig. 1 Pictorial view of the oblate coordinates in the plane $(v, \theta)$ with a hyperboloid and centered ellipsoid. It is shown a reduction of the oblate coordinates into a two dimensional plane with $\theta=0$. In this case, we have a two dimensional ellipsoid where $r \rightarrow 0$ is transformed into a singular ring (in the sense of Riemann invariants are infinite). In the case $r \rightarrow \infty$, the elliptical plane approaches to a circular plane

$$
\begin{aligned}
\left(\frac{d u}{d \phi}\right)^{2}= & u^{2}\left[\frac{3 a^{2} E^{2}}{e^{4 \sigma(u)} L^{2}}-1\right]+u^{4} a^{2} \beta^{2}\left[\frac{3 a^{2} E^{2}}{e^{4 \sigma(u)} L^{2}}-1\right] \\
& +a^{2} u^{4}\left[\frac{3 a^{2} E^{2}}{e^{4 \sigma(u)} L^{2}}-2\right]+\frac{\left(1+a^{2} u^{2} \beta^{2}\right) E^{2}}{e^{4 \sigma(u)} L^{2}}
\end{aligned}
$$

With the fact that the variable $u$ can be related with the oblate angles in such a way $r=a x=a \sinh v$, from Eq. (10), we can write $e^{-4 \sigma(v)}=e^{4 \beta \arctan (\operatorname{csch} v)}$. This allows us to study a closed positive infinite endpoints (asymptotic regions) of the orbit where $v=[0,+\infty]$. At $v \rightarrow+\infty$, the ellipsoid approaches to a circular orbit and at $v \rightarrow 0$ it approaches to a ring singularity [27], as illustrated in Fig. 1. Then elliptic trajectories can be studied inbetween from their respective endpoints, since the potential $\sigma$ does remain finite. Hence, using Eq. (10) and examining the tendencies, close to circular orbits with $v \rightarrow+\infty$, then $\sigma(v)$ approaches 0 , and the exponential term $e^{-4 \sigma(v)}$ approaches 1 . On the other hand, close to singularity, one can expand the related functions around zero $(v \rightarrow 0)$ of the argument of the exponential that leads to $-4 \sigma(v)=$ $-2 \beta \operatorname{sgn}(1 / v) \pi-v=-2 \beta \operatorname{sgn}(+\infty) \pi=-2 \beta \pi$, and the exponential term approaches $e^{-2 \beta \pi}$, where $s g n$ denotes the sign function. Thus, one can obtain two orbit equations in such a limits, respectively, 


$$
\begin{aligned}
\left(\frac{d u}{d \phi}\right)^{2}+u^{2}= & \frac{E^{2}}{L^{2}}+u^{2} \frac{a^{2} E^{2}}{L^{2}}\left(3+\beta^{2}\right) \\
& +u^{4} a^{2}\left(\beta^{2}\left[\frac{3 a^{2} E^{2}}{L^{2}}-1\right]+\left[\frac{3 a^{2} E^{2}}{L^{2}}-2\right]\right) \\
\left(\frac{d u}{d \phi}\right)^{2}+u^{2}= & \frac{E^{2}}{L^{2}} e^{-2 \beta \pi}+u^{2} \frac{a^{2} E^{2}}{L^{2}} e^{-2 \beta \pi}\left(3+\beta^{2}\right) \\
& +u^{4} a^{2}\left(\beta^{2}\left[\frac{3 a^{2} E^{2}}{L^{2}} e^{-2 \beta \pi}-1\right]\right. \\
& \left.+\left[\frac{3 a^{2} E^{2}}{L^{2}} e^{-2 \beta \pi}-2\right]\right)
\end{aligned}
$$

A good estimate of an effective orbit equation can be obtained by the asymptotic matched expansions given by the sum of Eqs. (25) and (26) and their difference with an "overlapped" orbit equation that results from setting $\beta=0$ in the two previous equation obtaining the same unique form. Hence, we can find the related orbit equation with singularityfree flat region in a form

$$
\left(\frac{d u}{d \phi}\right)^{2}+u^{2}=A+u^{2} B+u^{4} C=G(u),
$$

with $A, B$ and $C$ respectively

$$
\begin{aligned}
& A=\frac{E^{2}}{L^{2}} e^{-2 \beta \pi} . \\
& B=\frac{a^{2} E^{2}}{L^{2}}\left[\beta^{2}+\left(3+\beta^{2}\right) e^{-2 \beta \pi}\right],
\end{aligned}
$$

and

$$
\begin{aligned}
C= & a^{2} \beta^{2}\left[\frac{3 a^{2} E^{2}}{L^{2}}\left(1+e^{-2 \beta \pi}\right)-2\right] \\
& +a^{2}\left[\frac{3 a^{2} E^{2}}{L^{2}} e^{-2 \beta \pi}-2\right] .
\end{aligned}
$$

Using the method as shown in [5], we can work with the previous orbit equations analytically and the deviation angle $\delta \phi$ can be found using

$\delta \phi=\left.\pi \frac{d F(u)}{d u}\right|_{u_{0}}$,

with the constraint $F\left(u_{0}\right)=u_{0}$ for a near circular orbit. The function $F(u)$ is denoted by

$F(u)=\frac{1}{2} \frac{d G(u)}{d u}$

With those informations at hand, we can evaluate $F(u)$ straightforwardly

$F(u)=\frac{1}{2} \frac{d G(u)}{d u}=B u+2 u^{3} C$, and the related algebraic equation

$u_{0} B+2 u_{0}^{3} C=u_{0}$,

with solution

$u_{0}=\sqrt{\frac{1-B}{2 C}}$.

By using Eq. (31), it lead us to the "Zipoy's precession formula" given by the deviation angle

$\delta \phi_{(z i p)}=-2 \pi \frac{a^{2} E^{2}}{L^{2}}\left(3 e^{-2 \beta \pi}+\beta^{2}\left(1+e^{-2 \beta \pi}\right)\right)$.

Hence, we have an analytic relation in a flat space avoiding the asymptotic regions. Interestingly, besides the advanced solution, this formula also provides a retrograde precession in terms of the conserved quantities and initial parameters. It is noteworthy to point out that the hyperbolic term persists in the result evincing the propagation of the nonlinear effects from the Einstein equations even with the breakage of the diffeomorphic coordinate transformations.

To obtain the correct physical units, we use the known forms for the specific orbital energy $E=\frac{-G M}{2 \gamma}$ and the specific orbital momentum $L^{2}=\mu p$, with $\mu=G M$ and $p=l\left(1-\epsilon^{2}\right)$. The terms $M, l$ and $\epsilon$ denote the central Sun mass, the semi-major axis and the orbital eccentricity, respectively. The Newton's universal gravitational constant is denoted by $G$. Since $\beta$ is small, the hyperbolic exponential can be approximated to $e^{-2 \beta \pi} \sim 1-2 \beta \pi$. It is important to stress that high orders on $\beta$ are neglected. Accordingly, using Eq. (36), one can obtain

$\delta \phi_{(z i p)}=-\frac{3}{2} \pi \frac{a^{2} G M}{c^{2} l^{3}\left(1-\epsilon^{2}\right)}(1-2 \beta \pi)$.

A more familiar expression for apsidal precession can be obtained by using the orbital period $P$ in days in such a way we have the final form

$\delta \phi_{(z i p)}=\frac{-6 \pi^{3} a^{2}}{c^{2}\left(1-\epsilon^{2}\right) P^{2}}(1-2 \beta \pi)$,

In order to use physical measurements, we adopt the international system of measurement Bureau International des Poids et Mesures [74] setting one year $1 y r=365.256 d$, the speed of light $c=299,792,458 \mathrm{~m} / \mathrm{s}[26,74]$ and the mass of sun $M_{\odot}=1.98853 \times 10^{30} \mathrm{~kg}$. The period $P$ is given by $P=T(24)(3600)$ and $T$ is the sidereal orbital period in days. In the case of Mercury, we use $T=87.969$ days (NASA Mercury Fact Sheet. https://nssdc.gsfc.nasa.gov).

We use 9 data points concerning observations on the perihelion advance of Mercury in units of arcsecond per century $\left(\operatorname{arcsec} c y^{-1}\right)$ as shown in Table 1 . We denote $\delta \phi_{s c h}$ for standard (Einstein) perihelion precession and $\delta \phi_{W e y l}$ 
Table 1 Comparison between the values for secular precession of Mercury in units of arcsec/century(".cy ${ }^{-1}$ ) of the standard (Einstein) perihelion precession $\delta \phi_{s c h}$ [26] and the Weyl conformastatic solution $\delta \phi_{W e y l}$. The $\delta \phi_{o b s}$ stands for the secular observed perihelion precession in units of arcsec/century. In the fourth column, some observational values of perihelion precession are available. The first data point was adapted from [61] by adding a supplementary precession calibrated with the Ephemerides of the Planets and the Moon (EPM2011) [62,63]

\begin{tabular}{|c|c|c|c|c|}
\hline$\delta \phi_{s c h}$ & $\delta \phi_{W e y l}$ & $\delta \phi_{\text {Zipoy }}$ & $\delta \phi_{o b s}$ & References \\
\hline \multirow[t]{9}{*}{42.9781} & 43.105 & 42.9696 & $43.098 \pm 0.503$ & [61-63] \\
\hline & & & $43.20 \pm 0.86$ & [64] \\
\hline & & & $43.11 \pm 0.22$ & {$[65]$} \\
\hline & & & $43.11 \pm 0.22$ & [66] \\
\hline & & & $42.98 \pm 0.09$ & [67] \\
\hline & & & $43.13 \pm 0.14$ & {$[68]$} \\
\hline & & & $42.98 \pm 0.04$ & {$[69,70]$} \\
\hline & & & $43.03 \pm 0.00$ & [71] \\
\hline & & & $43.11 \pm 0.45$ & {$[72,73]$} \\
\hline
\end{tabular}

for the resulting perihelion advance using the Weyl conformastatic solution [40,76], which comes from an axiallysymmetric motion of a test particle in Weyl's line element [31]. To control the systematics, we use GnuPlot 5.2 software to compute non-linear least-squared fitting by using the Levenberg-Marquardt algorithm for the goodness-of-fitting to data. From this algorithm, we obtain the values for the parameters and the related reduced chi-squared $\left(\chi_{\text {red }}^{2}\right)$. Since Eq. (38) has a negative sign, and to obtain an advanced precession solution, we calculate its absolute value. We observed that running the parameters freely, we find that the $a$ parameter has the same magnitude of the planetary semi-major axis as it provides $a \sim-1.15806 \times 10^{11}$, which its absolute value is roughly close to observational value of Mercury's semi-major axis and $\beta=8.86038 \times 10^{-6}$ and the resulting value for the shift angle is $42.9696 \operatorname{arcsec} c y^{-1}$ for a $\chi_{\text {red }}^{2}=0.0166$ and a probability $p>0.95$, which represents a good fitting. It is worth noting that the negative sign for the length parameter $a$ is a relic from the hyperbolic geometry that passed through the nonlinear effects of the initially strong gravitational field. Interestingly, the lower values of $\beta$ indicates a Schwarzschild-like integrable system of the inbetween studied zone [75] which implies that such zone is an island of stability.

In Table 1, we show the secular precession of Mercury in units of arcsec/century comparing with standard result of Schwarzschild solution and the cylindric Weyl solution for the perihelion shift. The obtained perihelion shift $\delta \phi_{\text {Zipoy }}$ reproduces closely the observed perihelion shift with a bonus that it naturally provides elliptical orbits which makes this solution a better physical description for astrophysical purposes according to the shape, the topology and the symmetry
Table 2 Comparison between the observational values $\delta \phi_{o b s}$ for secular precession in units of arcsec/century and the values from the standard (Einstein) perihelion precession and the Zipoy solution $\delta \phi_{\text {model }}$ for selected asteroid 1566 Icarus and 2-Pallas

\begin{tabular}{lclc}
\hline Object & $\delta \phi_{\text {obs }}\left({ }^{\prime \prime} . c y^{-1}\right)$ & $\delta \phi_{\text {sch }}\left({ }^{\prime \prime} . c y^{-1}\right)$ & $\delta \phi_{\text {model }}\left(" . c y^{-1}\right)$ \\
\hline 1566 Icarus & 10.05 & 10.0613 & 10.029 \\
2 Pallas & -133.534 & - & -133.52 \\
\hline
\end{tabular}

aspects of the gravitational field. An interesting case relies on the asteroids astrodynamics.

Departing from a spherical geometry, we are able to study precession of two asteroids as shown in Table 2. The first one corresponds to the asteroid Icarus. This asteroid is a nearEarth object (NEO) of the Apollo group with a very elliptical orbit. It has been regarded as a relativistic asteroid with an approximation even close to the Sun than Mercury and also a Venus and Mars-crosser. Its observational value for the perihelion precession is 10.05 arcseconds per century with semimajor axis $1.61258 \times 10^{11} \mathrm{~m}$ and a large eccentricity 0.82695 for an orbital period $T=408.781$ days [26]. As a result, we obtained the values for the parameters $a \sim-3.21987 \times 10^{11}$ and $\beta=8.0222 \times 10^{-6}$ that provide a value for the shift angle $10.029 \operatorname{arcsec} c y^{-1}$ for $\chi_{\text {red }}^{2}=0.00272$ and $p>0.95$.

In addition, as an example of retrograde precession, which is not accounted for the standard Einstein perihelion formula, we studied the 2-Pallas protoplanet, even though the available informations on 2-Pallas are still scarce. The 2-Pallas asteroid is one of the largest asteroids in asteroid belt and is a Jupiter-crosser. Its observational value for the perihelion precession is -1333.534 arcseconds per century with semimajor axis $4.14520 \times 10^{11} \mathrm{~m}$ and a large eccentricity 0.2812 for an orbital period $T=1686.43$ days (available at https:// newton.spacedys.com/astdys $2 /$ index.php?pc $=3.0$, Asteroids Dynamic Site-AstDyS). As a result, we obtained the values for the parameters $a \sim-1.680 \times 10^{13}$ and $\beta=8.0222 \times$ $10^{-6}$ that provide a value for the shift angle $-133.481 \mathrm{arcsec}$ $c y^{-1}$ for $\chi_{\text {red }}^{2}=1245.46$ and $p>0.95$. In the two previous cases, the value of the $\beta$ parameter remains the same and unless we find a counterproof in the near future, its value around $\sim 10^{-6}$ must remain the same for any large object in Solar system (large asteroid, comets and planets). As shown, the produced gravitational field in this space-time is not the same as the Schwarzschild case. In this case, the Zipoy spacetime seems to be more astrophysically adapted as compared to the standard PPN solutions and it naturally provides both advance and retrograde precessions.

\section{Final remarks}

Our results in this paper constitute a fine example that the non-linearities of a system of equations imprint qualitative 
effects on the orbits of their solutions. We have studied solutions of vacuum Einstein's equation of a quasi-oblate metric obtaining a set of solutions that depends on the Legendre Polynomials, based on Zipoy's seminal paper [27]. In hindsight, the simplest studied case was the so-called "monopole" solution for the zeroth order of Legendre polynomials $l=0$. Starting from the related Lagrange equations, we have obtained the orbit equations in the asymptotic regions, which revealed to be a highly nonlinear set of equations. To obtain an analytical solution, we have studied a closed positive infinite interval to get an elliptical pattern of the orbits in-between in a flat space. As a result, we have obtained a non-standard formula for the perihelion precession depending on the dimensionless parameter $\beta$ and the length parameter $a$. The $\beta$ parameter was primarily fixed as a low magnitude to allow us to study the orbit equation and latter it was be found to be of the order of $\sim 10^{-6}$. In terms of the $\gamma$ metric, it is compatible with the condition for an integrable system with $\gamma \longrightarrow 1$.

It is worth noting to point out that no a priori assumptions concerning the strength of the field (as a weak field) were imposed. Moreover, the values of the length parameter $a$ were adjusted numerically using the Chi-squared statistics for 9 observational datasets. We have shown that, as pointed out by Zipoy, the length parameter can be attributed to a physical meaning since it is closely related to semi-major axis. Interestingly, the values converged to the same order of magnitude of semi-major axis of Mercury. Differently from the standard Einstein's solution and the Weyl cylindrical one, the precession formula from oblate coordinates provides naturally both retrograde and advanced solutions for the perihelion precession besides the fact that elliptical orbits are also native in those coordinates, which reinforces the idea that the topological nature of the problem is now an important character and the strength of the gravitational field is highly constrained by this topology. In summary, this analysis was made in the realm of GR in a nearly newtonian limit with no need of additional extensions or modifications of the standard gravity. As future perspectives, the extended analysis of the deviation of light, radar echo and gravitational lens in the oblate metric are currently in progress.

Acknowledgements Paola T.Z. Seidel thanks the Coordination for the Improvement of Higher education Personnel (Capes-Brazil) and the Fundação Araucária/PR for the scholarship grant Capes/FA (Chamada Pública 19/2015) and A. J. S. Capistrano thanks Federal University of Latin-American Integration for financial support from Edital PRPPG $\mathrm{n}^{\circ}$ 110 (17/09/2018) and Fundação Araucária/PR for the Grant CP15/2017. P\&D. L. A. Cabral thanks the APGP-UNILA for kind support. We also thank Carlos Coimbra and Rodrigo Bloot for fruitful discussions and criticisms.

Data Availability Statement This manuscript has no associated data or the data will not be deposited. [Authors' comment: All the used data are provided in the related references. Concerning minor objects, the used data is publicly provided at https://newton.spacedys.com/astdys $2 /$ index.php?pc=3.0, Asteroids Dynamic Site-AstDyS.]

Open Access This article is distributed under the terms of the Creative Commons Attribution 4.0 International License (http://creativecomm ons.org/licenses/by/4.0/), which permits unrestricted use, distribution, and reproduction in any medium, provided you give appropriate credit to the original author(s) and the source, provide a link to the Creative Commons license, and indicate if changes were made.

Funded by SCOAP ${ }^{3}$.

\section{References}

1. S. Chekanov et al. (ZEUS Collaboration), Eur. Phys. J. C 42, 1 (2005)

2. H.J. Schmidt, Phys. Rev. D 78, 023512 (2008)

3. P.H. Lim, P.S. Wesson, Astrophys. J. 397, L91-L94 (1992)

4. L. Iorio, Sch. Res. Exch. 2008, 238385 (2008)

5. T. Harko, Z. Kovács, F.S.N.Lobo, Proc. Roy. Soc. Lond. A Math. Phys. Eng. Sci. 467, 1390-1407 (2011)

6. M.K. Mak, T. Harko, Phys. Rev. D 70, 024010 (2004)

7. M.D. Maia, A.J.S. Capistrano, D. Muller, Int. J. Mod. Phys. 18, 1273-1289 (2009)

8. Y.K.E. Cheung, F. Xu, Astrophys.J. 774, 1, 65, 20 (2013)

9. S. Chakraborty, S. Sengupta, Phys. Rev. D 89, 026003 (2014)

10. S. Jalalzadeh, M. Mehrnia, H.R. Sepangi, Class. Quantum Gravity 26, 155007 (2009)

11. L. Iorio, A.J. 137, 3615 (2009)

12. L. Iorio, Int. J. Mod. Phys. D 18, 947 (2009)

13. A. Avalos-Vargas, G. Ares de Parga, EPJ Plus 127, 155 (2012)

14. H. Arakida, Int. J. Theor. Phys. 52(5), 1408-1414 (2013)

15. G.S. Adkins, J. McDonnell, Phys. Rev. D 75(8), 082001 (2007)

16. A. Biswas, K. Mani, Cent. Eur. J. Phys. 3(1), 69-76 (2005)

17. M.M. D'Eliseo, Astrophys. Sp. Sci. 342(1), 15-18 (2012)

18. X. Deng, Y. Xie, Astrophys. Sp. Sci. 350(1), 103-107 (2014)

19. M.R. Feldman, PLoS ONE 8(11), e78114 (2013)

20. Z. Li, S.-F. Yuan, C. Lu, Y. Xie, Res. Astron. Astrophys. 14, 2 , 139-143 (2014)

21. L. Iorio, Class. Quantum Gravity 22(24), 5271-5281 (2005)

22. L. Iorio, JCAP 05, 002 (2006)

23. L. Iorio, Adv. Astron. 2008(268647), 1-5 (2008)

24. L. Iorio, H.I.M. Lichtenegger, M.L. Ruggiero, C. Corda, Astrophys. Sp. Sci. 331(2), 351-395 (2011)

25. M.L. Ruggiero, Int. J. Mod. Phys. D 23(5), 1450049 (2014)

26. K. Wilhelm, B.N. Dwivedi, New Astron. 31, 51-55 (2014)

27. M.D. Zipoy, J. Math. Phys. 7, 1137 (1966)

28. C. Misner, K.S. Thorne, J.A. Wheeler, Gravitation (W.H. Freeeman \& Co., Reading, 1973)

29. L. Infeld, J. Plebanski, Motion and Relativity (Pergamon Press, New York, 1960)

30. A.J.S. Capistrano, Galaxies 6, 48 (2018)

31. H. Weyl, Ann. Phys. 359, 117 (1917)

32. N. Rosen, Rev. Mod. Phys. 21, 503 (1949)

33. R. Gautreau, R.B. Hoffman, A. Armenti, Il Nuovo Cimento B 7(1), 71-98 (1972)

34. H. Stephani et al., Exact Solutions of Einstein's Field Equations (Cambridge University Press, Cambridge, 2003)

35. G.A. González, A.C. Gutiérrez-Piñeres, P.A. Ospina, Phys. Rev. D 78, 064058 (2008)

36. A.C. Gutiérrez-Piñeres, G.A. González, H. Quevedo, Phys. Rev. D 87, 044010 (2013)

37. M. Ujevic, P.S. Letelier, Phys. Rev. D 70, 084015 (2004)

38. M. Ujevic, P.S. Letelier, Gen. Relativ. Gravit. 39, 1345-1365 (2007) 
39. D. Vogt, P.S. Letelier, Mon. Not. R. Astron. Soc. 384, 834-842 (2008)

40. A.J.S. Capistrano, W.L. Roque, R.S. Valada, Mon. Not. R. Astron. Soc. 444, 1639-1646 (2014)

41. C.M. Will, Phys. Rev. Lett. 120, 191101 (2018)

42. G. Erez, N. Rosen, Bull. Res. Counc. Israel 8, 47 (1959)

43. Y.A.B. Zeldovich, I.D. Novikov, Relativistic Astrophysics (University of Chicago Press, Chicago, 1971)

44. H. Quevedo, Phys. Rev. D 39, 2904 (1989)

45. B.H. Voorhees, Phys. Rev. D 2, 2119 (1970)

46. H. Kodama, W. Hikida, Class. Quantum Gravity 20, 5121-5140 (2003)

47. A.M. Sherif, Computing the Arnold Tongue in the Zipoy-Voorhees Space-time, Master Thesis, University of Stellenbosch-SA (2017)

48. G.W. Gibbons, M.S. Volkov, JCAP 05(2017), 039 (2017)

49. A. Tomimatsu, H. Sato, Phys. Rev. Lett. 29, 1344 (1972)

50. A. Tomimatsu, H. Sato, Prog. Theor. Phys. 50, 95 (1973)

51. G.W. Gibbons, M.S. Volkov, Phys. Lett. B 760, 324-328 (2017)

52. S. Parnovsky, Zh Eksp, Teor. Fiz. 88, 1921 (1985)

53. S. Parnovsky, JETP 61, 1139 (1985)

54. D. Papadopoulos, B. Stewart, L. Witten, Phys. Rev. D 24, 320 (1981)

55. L. Herrera, J.L. Hernandez-Pastora, J. Math. Phys. 41, 7544 (2000)

56. L. Herrera, G. Magli, D. Malafarina, Gen. Relativ. Gravit. 37, 1371 (2005)

57. A.N. Chowdhury, M. Patil, D. Malafarina, P.S. Joshi, Phys. Rev. D 85, 104031 (2012)

58. K. Boshkayev et al., Phys. Rev. D 93(2), 024024 (2016)

59. B. Toshmatov, D. Malafarina, N. Dadhich, Phys. Rev. D 100(4), 044001 (2019)
60. K. Yamada, H. Asada, Mon. Not. R. Astron. Soc. 423, 3540-3544 (2012)

61. G.G. Nambuya, Mon. Not. R. Astron. Soc. 403, 1381 (2010)

62. E.V. Pitjeva, N.P. Pitjev, Mon. Not. R. Astron. Soc. 432(4), 34313437 (2013)

63. N.P. Pitjev, E.V. Pitjeva, Astron. Lett. 39(3), 141-149 (2013)

64. I.I. Shapiro et al., Phys. Rev. Lett. 28, 1594-1597 (1972)

65. I.I. Shapiro, C.C. Counselman III, R.W. King, Phys. Rev. Lett. 36, 555-585 (1976)

66. J.D. Anderson et al., Acta Astronaut. 5, 43-61 (1978)

67. I.I. Shapiro, Solar system tests of general relativity: recent results and present plan, In: N. Ashby et al (Eds.): General Relativity and Gravitation, 1989, Proceedings of the 12th International Conference on General Relativity and Gravitation, Cambridge: Cambridge University Press, 313-330 (1990)

68. J.D. Anderson et al., Recent developments in solar-system tests of general relativity, in Proceedings of the 6th Marcel Grossmann Meeting on General Relativity, Kyoto, June 1991, eds. H. Sato et al. (World Scientific, Singapore, 1992), p. 353-355

69. A.M. Nobili, C.M. Will, Nature 320, 39-41 (1986)

70. C.M. Will, Living Rev. Relativ. 9, 3 (2006)

71. G.M. Clemence, Icarus 3, 502 (1964)

72. R.L. Duncombe, Astron. J. 61, 174-175 (1956)

73. D.C. Morton, J.R. Astron, Soc. Can. 50, 223 (1956)

74. Bureau International des Poids et Mesures, Le Systéme International d'Unités(SI), 8e édition, BIPM, Sévres, p. 37 (2006)

75. G. Lukes-Gerakopoulos, Phys. Rev. D 86, 044013 (2012)

76. A.J.S. Capistrano, J.A.M. Penagos, M.S. Alárcon, Mon. Not. R. Astron. Soc. 463, 1587-1591 (2016) 